\title{
An interior-point algorithm for computing equilibria in economies with incomplete asset markets
}

\author{
M. Esteban-Bravo* \\ Department of Business Administration, Universidad Carlos III de Madrid, C/Madrid, 126. 28903 Getafe, \\ Madrid, Spain \\ Received 4 September 2003; accepted 2 February 2007 \\ Available online 14 April 2007

\begin{abstract}
Computing equilibria in general equilibria models with incomplete asset (GEI) markets is technically difficult. The standard numerical methods for computing these equilibria are based on homotopy methods. Despite recent advances in computational economics, much more can be done to enlarge the catalog of techniques for computing GEI equilibria. This paper presents an interior point algorithm that exploits the special structure of GEI markets. It is proved that, under mild conditions, the algorithm converges globally at a quadratic rate, rendering it particularly effective in solving large scale GEI economies. To illustrate its performance, relevant examples of GEI markets are solved.
\end{abstract}

JEL classification: C68; C63

Keywords: General equilibrium; Incomplete markets; Computation of equilibria; Interior point methods

\section{Introduction}

Since the time of Radner's (1972) paper, the general equilibrium analysis of sequential economies with incomplete markets has been the subject of extensive

\footnotetext{
*Tel.: + 34916248921 ; fax: + 34916249607 .

E mail address: mesteban@emp.uc3m.es.
} 
research in economic theory. These models, known as general equilibrium with incomplete markets (GEI), represent the most general and versatile tool in competitive economic theory, as they deal simultaneously with all real and financial markets and their interactions. The size and complexity of these models often demands the use of numerical methods to compute their equilibria. Surveys in this area are due to Magill and Shafer (1991), Magill and Quinzii (1996) and Hens (1998).

The literature on the computation of GEI equilibria is based on path following or homotopy methods. There are three main approaches to compute equilibria in the GEI model. The first method was given by DeMarzo and Eaves (1996), who considered the excess demand function defined on prices and elements of the Grassmannian manifold (see e.g. Duffie and Shafer, 1985). By applying the work of Brown et al. (1996a), they computed equilibria via a homotopy algorithm. The second algorithm for computing fixed points was developed by Brown et al. (1996b), who considered the excess demand function to be a function of prices only. Because this excess demand function is discontinuous, Brown et al. introduced an auxiliary asset to define a family of homotopies. The third approach is based on the work of Schmedders $(1998,1999)$, who computed equilibria with homotopy techniques using the first-order conditions of the no-arbitrage agents' problems. To avoid discontinuities in the excess demand correspondence, he considered one agent with penalties for transactions on the asset markets instead of assuming lower bounds on short sales. By making these penalties smaller and smaller, the solutions of the homotopy function move closer and closer to the GEI equilibrium. Other contributions of note are those of Kubler and Schmedders (2000), Kubler (2001) and Herings and Kubler (2002).

Homotopy methods possess powerful theoretical properties, but these methods may be inappropriate from a practical point of view, due to the difficulty of setting up the homotopy function (see Kubler, 2001) and dealing with inequalities (see Watson, 2000). Furthermore, homotopy methods may fail to produce a solution even for relatively simple systems of nonlinear equations (e.g.. see Nocedal and Wright, 1999, Example 11.2). The focus of this paper is a proposal for an alternative algorithm for problems of practical relevance that cannot be solved by the existing approaches.

This paper introduces a tailor-made interior-point algorithm to compute equilibria in economies with incomplete asset markets. I consider a set of optimality conditions which are necessary and sufficient conditions for the existence of a GEI equilibrium, assuming the standard convexity assumptions for the agents' problem. These optimality conditions are the Karush-Kuhn-Tucker (KKT) first-order conditions of the agents' utility maximization problems, the market clearing and no-arbitrage conditions. A distinctive characteristic in solving these optimality conditions is that they often include inequalities (e.g. equilibria can be restricted-domain), which can be difficult to handle using homotopy or continuation methods. I propose an alternative interior-point approach which is tailored to deal effectively with inequality-constrained nonlinear systems of equations.

Although interior-point methods are closely related to central path continuation methods (see Gill et al., 1986), the solution procedures are completely different. 
A relevant property of interior-point methods is their typical requirement for significantly less function and derivative evaluations and linear algebra operations than are required by homotopy methods (see Garcia and Zangwill, 1981). This feature makes interior-point algorithms an attractive alternative to homotopy when considering large-scale GEI models such as the pricing of financial assets. In a recent survey, Esteban-Bravo (2004) suggests the application of interior-point methods to compute equilibria in complete markets. In this paper I fully explore this approach for solving GEI models, the complexity and scale of which demand efficient algorithms to compute equilibria.

The proposed interior-point algorithm enjoys some computational advantages over the standard algorithms. In particular, I introduce two devices for increasing the speed of computations. Following the Gauss-Newton arguments, the secondorder information of the nonlinear elements of the problem is neglected, a strategy that reduces the number of function evaluations needed. Second, the sparsity properties of GEI models is exploited. As a consequence, the algorithm finds accurate solutions with less computational costs than does a standard interiorpoint method. To be rigorous, I prove that, under mild conditions, the algorithm globally converges to KKT points at a quadratic rate (i.e. that the algorithm will eventually find an equilibrium assuming that the economy has any and the required conditions hold).

Computational examples are presented, documenting the fact that the implementation is capable of solving relevant examples of GEI markets robustly and efficiently. Even though numerical comparisons lie beyond the scope of this paper, I compute equilibria for GEI models considered by Schmedders (1998), showing competitive running times. The numerical results are very encouraging, showing computational gains when applying to large-scale models, and that this algorithm is particularly suited to solving problems in which some or all of the variables of interest are bounded. Such problems are commonly found in the literature of incomplete markets, for example, when considering short-selling constraints. Furthermore, I illustrate that the algorithm diverges when it is considered economies in which equilibria do not exist.

The remainder of the paper is organized as follows. Section 2 presents a twoperiod general equilibrium model with incomplete asset markets and the characterization of the equilibrium as the first-order conditions of the agents' utility maximization problems, the market clearing, and no-arbitrage conditions. In Section 3, I present an interior-point algorithm to compute such an equilibrium and study its convergence properties. Finally, the algorithm has been implemented for relevant examples of GEI markets, illustrating its potential for application in many problems, as shown in Section 4.

\section{The GEI model}

The basic GEI model describes an exchange economy over two time periods $(t=0,1)$, with uncertainty over the state of nature in Period 1 . At time $t=0$ the 
economy is in state $s=0$ which is known by each of the $I$ consumers participating in the economy (i.e. all relevant information is symmetric across the economy). However, it is not known which of the $S$ possible states at time $t=1$ will occur. Trade occurs sequentially over time.

Assume that the markets on which commodities and financial assets are traded are competitive. In each state there are $D$ goods, and for each good $d$ in state $s$ there exists a spot market with spot price $p_{s d}$. Hence, the commodity space is $\mathbb{R}_{+}^{D(S+1)}$. For any $x \in \mathbb{R}_{+}^{D(S+1)}, x^{\mathrm{T}}$ denotes the transpose of $x$, a $D(S+1)$-dimensional row vector. For any $x, y \in \mathbb{R}_{+}^{D(S+1)}, x \cdot y=x^{\mathrm{T}} y$ denotes the inner product of vectors $x$ and $y$. There is a finite number $C$ of assets traded on financial markets. Let $\theta_{i} \in \mathbb{R}^{C}$ denote the portfolio of traded assets by the $i$ th consumer. An asset $c$ can be purchased for a price $q_{c}$ at time $t=0$, and delivers a return across the states at time $t=1$. The return of an asset $c$ is described by its asset matrix $A^{c}=\left(A_{1}^{c}, \ldots, A_{S}^{c}\right)^{\mathrm{T}}$, defined across all states at time $t=1$, where $A_{s}^{c}$ is the commodity bundle which asset $c$ delivers for state $s$. The matrix $A^{c}$ can be specified exogenously or be given as a function of some variables observed at $t=1$. The asset $c$ delivers a nominal return $V_{s}^{c}=p_{s} \cdot A_{s}^{c}$ in state $s$. Therefore, the asset structure is summarized by the asset matrix $A$ (given in units of commodities) and the nominal return matrix $V(p)$ :

$$
\underset{D S \times C}{A}=\left(\begin{array}{lll}
A_{11}^{1} & \ldots & A_{11}^{C} \\
\vdots & & \vdots \\
A_{S D}^{1} & \ldots & A_{S D}^{C}
\end{array}\right), \quad \underset{S \times C}{V(p)}=\left(\begin{array}{lll}
V_{1}^{1}\left(p_{1}\right) & \ldots & V_{1}^{C}\left(p_{1}\right) \\
\vdots & & \vdots \\
V_{S}^{1}\left(p_{S}\right) & \ldots & V_{S}^{C}\left(p_{S}\right)
\end{array}\right) .
$$

Assume that $S \geqslant C$. The completeness condition is important in the context of GEI markets. The financial markets are said to be complete if $\operatorname{rank}(V(p))=S$ for any $p$. Under this condition, agents can insure themselves against any type of contingency in period $t=1$. When $\operatorname{rank}(V(p))<S$, the financial markets are said to be incomplete (see Magill and Shafer, 1991).

Each consumer is described by a consumption set $\mathbb{R}_{+}^{D(S+1)}$, initial endowments for the $D(S+1)$ goods in each state $w_{i}=\left(w_{i 0}, w_{i 1}, \ldots, w_{i S}\right) \in \mathbb{R}_{+}^{D(S+1)}$, and a preference relation. The utility function $u_{i}: \mathbb{R}_{+}^{D(S+1)} \longrightarrow \mathbb{R}$ that represents the $i$ th consumer's preferences is assumed to be continuously differentiable, concave, strictly monotonous and increasing. Given $p \in \mathbb{R}_{+}^{D(S+1)}$ and $q \in \mathbb{R}^{C}$, each consumer faces the following problem:

$$
\begin{aligned}
\max _{x, \theta} & u_{i}\left(x_{i 0}, \ldots, x_{i S}\right) \\
\text { s.t. } & p_{0} \cdot x_{i 0} \leqslant p_{0} \cdot w_{i 0}-q \cdot \theta_{i}, \\
& p_{s} \cdot x_{i s} \leqslant p_{s} \cdot w_{i s}+p_{s} \cdot A_{s} \theta_{i}, \quad \forall s .
\end{aligned}
$$

An equilibrium for the GEI economy is defined to be the prices $\left(p^{*}, q^{*}\right)$ and the allocation $\left(x^{*}, \theta^{*}\right)$ satisfying: (i) $\left(x_{i}^{*}, \theta_{i}^{*}\right)$ is an optimal solution to Problem (1), $\forall i=1, \ldots, I$; (ii) $\sum_{i=1}^{I} x_{i}^{*}=\sum_{i=1}^{I} w_{i}$, and $\sum_{i=1}^{I} \theta_{i}^{*}=0$.

The next theorem provides a characterization of GEI equilibria. 
Theorem 1 (Characterization of GEI equilibria). Assume that $A_{s}$ has full rank, i.e. $\operatorname{rank}\left(A_{s}\right)=C, \forall s$. The allocation $\left(x^{*}, \theta^{*}, p^{*}, q^{*}\right)$ is an equilibrium for the economy if and only if there exist $\delta^{*} \in \mathbb{R}_{++}^{I(S+1)}$ and $\left(x^{*}, \theta^{*}, p^{*}, q^{*}\right) \in \mathbb{R}_{+}^{I D(S+1)} \times \mathbb{R}^{I C} \times \mathbb{R}_{++}^{D(S+1)} \times$ $\mathbb{R}^{C}$ that satisfies the following optimality conditions:

$$
\begin{aligned}
& \begin{cases}\delta_{i s}^{*} \nabla_{x_{i s}} u_{i}\left(x_{i}^{*}\right)-p_{s}^{* T}=0, & \forall i, \forall s, \\
p_{0}^{* T}\left(x_{i 0}^{*}-w_{i 0}\right)+q^{* T} \theta_{i}^{*}=0, & \forall i, \\
p_{s}^{* T}\left(x_{i s}^{*}-w_{i s}-A_{s} \theta_{i}^{*}\right)=0, & \forall i, \forall s,\end{cases} \\
& \sum_{s=1}^{S} \frac{\delta_{i 0}^{*}}{\delta_{i s}^{*}} p_{s}^{*} A_{s}-q^{*}=0, \quad \forall i, \\
& \left\{\begin{array}{l}
\sum_{i=1}^{I} x_{i}^{*}=\sum_{i=1}^{I} w_{i}, \\
\sum_{i=1}^{I} \theta_{i}^{*}=0 .
\end{array}\right.
\end{aligned}
$$

Eq. (3) implies that in equilibrium the asset prices $q^{*}$ do not allow arbitragenamely that there exists a $\beta \in \mathbb{R}_{++}^{S}$ so that $\sum_{s=1}^{S} \beta_{s} p_{s}^{*} A_{s}-q^{*}=0$.

\section{An interior-point algorithm}

In this section I present an interior-point algorithm to compute GEI equilibria using the characterization given in Theorem 1 . To facilitate the exposition, $H(z)=0$ denotes the system of nonlinear equations given by (2)-(4), where $z=(\delta, x, \theta, p, q)$ $\in \mathbb{R}_{++}^{I(S+1)} \times \mathbb{R}_{+}^{I D(S+1)} \times \mathbb{R}^{I C} \times \mathbb{R}_{++}^{D(S+1)} \times \mathbb{R}^{C}$.

The system $H(z)=0$ is first reformulated as a least-squares problem and then, logarithmic barrier terms are introduced in order to remove the non-negativity bounds. The result is the following unconstrained barrier problem:

$$
\min \frac{1}{2}\|H(z)\|_{2}^{2}-\mu(\log \delta+\log x+\log p),
$$

where $\log \delta=\sum_{i=1}^{I} \sum_{s=0}^{S} \log \delta_{i s}, \log x=\sum_{i=1}^{I} \sum_{d=1}^{D} \sum_{s=0}^{S} \log x_{i d s}$, and $\log p=\sum_{d=1}^{D}$ $\sum_{s=0}^{S} \log p_{d s}$, with $(\delta, x, p)>0$, and $\mu>0$ is a scalar called the barrier parameter. By letting $\mu$ converge to zero, the sequence of solutions $\left\{z_{\mu}^{*}\right\}$ to (5) converges to a solution $z^{*}$ of $\min \|H(z)\|_{2}^{2}$. I also consider a tolerance parameter $\varepsilon_{\text {SIZE }}$, which forces the solutions $\left\{z_{\mu}^{*}\right\}$ to satisfy $\left\|H\left(z_{\mu}^{*}\right)\right\|_{2}^{2} \leqslant \varepsilon_{\text {SIZE }}$. To ensure that the local minimizer $z^{*}$ is a solution of the original nonlinear system $H\left(z^{*}\right)=0$, the barrier parameter is decreased from one barrier problem to the next and must converge to zero. Therefore, I aim to compute the sequence of solutions to (5) with $\left\|H\left(z_{\mu}^{*}\right)\right\|_{2}^{2} \leqslant \varepsilon_{\text {SIZE }}$. 
A minimizer for Problem (5 must satisfy the perturbed KKT conditions:

$$
J(z)^{\mathrm{T}} H(z)-\mu \Sigma^{1} e=0,
$$

where $J(z)$ denotes the Jacobian matrix of $H(z) ; \Sigma=\operatorname{diag}\left(\delta, x, 0_{I C \times 1}, p, 0_{C \times 1}\right)$ defines a diagonal matrix, the diagonal entries of which are the components of vector $\left(\delta, x, 0_{I C \times 1}, p, 0_{C \times 1}\right)$, where $0_{I C \times 1}$ denotes the vector of $I C$ zeros; and $\Sigma^{1}$ is the pseudo-inverse matrix of $\Sigma$. Let $e=(1, \ldots, 1)^{\mathrm{T}}$ denote the vector of ones, then $\Sigma e$ defines the vector $\left(\delta, x, 0_{I C \times 1}, p, 0_{C \times 1}\right)$ and $\Sigma^{1} e$ denotes the vector $\left(1 / \delta, 1 / x, 0_{\text {IC } \times 1}, 1 / p, 0_{C \times 1}\right)$.

Introducing an auxiliary variable $w$, so that $w=\left(w^{\delta}, w^{x}, 0_{I C \times 1}, w^{p}, 0_{C \times 1}\right) \in$ $\mathbb{R}_{+}^{I(S+1)+I D(S+1)+I C+D(S+1)+C}$, with $w^{\delta}, w^{x}, w^{p}>0$, and $w=\mu \Sigma{ }^{1} e$; the KKT conditions can be rewritten as follows:

$$
\begin{aligned}
& J(z)^{\mathrm{T}} H(z)-w=0, \\
& \operatorname{diag}(\delta) w^{\delta}-\mu e=0, \\
& \operatorname{diag}(x) w^{x}-\mu e=0, \\
& \operatorname{diag}(p) w^{p}-\mu e=0,
\end{aligned}
$$

where $\operatorname{diag}(\delta), \operatorname{diag}(x)$, and $\operatorname{diag}(p)$ define the diagonal matrices of vectors $\delta, x, p$, respectively. Note that the nonlinear transformation $w=\mu \Sigma^{1} e$ is only considered for the bounded variables $(\delta, x, p)>0$, and therefore $\left(w^{\delta}, w^{x}, w^{p}\right) \in$ $\mathbb{R}_{++}^{I(S+1)+I D(S+1)+D(S+1)}$ as $\mu>0$.

In essence, an interior-point method consists of the application of Newton's method to find a solution to the nonlinear system (6). Newton's method provides search directions $\left(\Delta z, \Delta w^{\delta}, \Delta w^{x}, \Delta w^{p}\right)$ from the first-order Taylor series expansion for (6) about the values $\left(z, w^{\delta}, w^{x}, w^{p}\right)$. Let $\left(z_{k}, w_{k}^{\delta}, w_{k}^{x}, w_{k}^{p}\right)$ be the current iterate. Then the search direction $\left(\Delta z, \Delta w^{\delta}, \Delta w^{x}, \Delta w^{p}\right)$ is the solution of the following system of linear equations:

$$
\left(\begin{array}{cc}
J_{k}^{\mathrm{T}} J_{k}+L_{k} H_{k} & -I \\
\operatorname{diag}\left(w_{k}^{\delta}, w_{k}^{x}, w_{k}^{p}\right) & \operatorname{diag}\left(\delta_{k}, x_{k}, p_{k}\right)
\end{array}\right)\left(\begin{array}{c}
\Delta z \\
\Delta w^{\delta} \\
\Delta w^{x} \\
\Delta w^{p}
\end{array}\right)=-\left(\begin{array}{c}
J_{k}^{\mathrm{T}} H_{k}-w_{k} \\
\operatorname{diag}\left(\delta_{k}\right) w_{k}^{\delta}-\mu e \\
\operatorname{diag}\left(x_{k}\right) w_{k}^{x}-\mu e \\
\operatorname{diag}\left(p_{k}\right) w_{k}^{p}-\mu e
\end{array}\right),
$$

where $H_{k}=H\left(z_{k}\right)$ denotes the system $H$ evaluated at $\left(z_{k}, w_{k}^{\delta}, w_{k}^{x}, w_{k}^{p}\right), J_{k}=J\left(z_{k}\right)$ denotes Jacobian of $H_{k}$ evaluated at $\left(z_{k}, w_{k}^{\delta}, w_{k}^{x}, w_{k}^{p}\right)$, and $L_{k}$ denotes the Hessian of $H$ evaluated at $\left(z_{k}, w_{k}^{\delta}, w_{k}^{x}, w_{k}^{p}\right)$. Note that $\Delta z$ denotes the search direction $(\Delta \delta, \Delta x, \Delta \theta, \Delta p, \Delta q)$.

The next iterate $\left(z_{k+1}, w_{k+1}^{\delta}, w_{k+1}^{x}, w_{k+1}^{p}\right)$ is defined as $\left(z_{k}, w_{k}^{\delta}, w_{k}^{x}, w_{k}^{p}\right)+\left(\Delta z, \Delta w_{k}^{\delta}\right.$, $\left.\Delta w_{k}^{x}, \Delta w_{k}^{p}\right)$. However, rather than solving each system (7) as the standard interiorpoint method would do, I am content with an approximate solution $\left(z_{k}, w_{k}^{\delta}, w_{k}^{x}, w_{k}^{p}\right)$ 
satisfying

$$
\left(\begin{array}{cc}
J_{k}^{\mathrm{T}} J_{k} & -I \\
\operatorname{diag}\left(w_{k}^{\delta}, w_{k}^{x}, w_{k}^{p}\right) & \operatorname{diag}\left(\delta_{k}, x_{k}, p_{k}\right)
\end{array}\right)\left(\begin{array}{c}
\Delta z \\
\Delta w^{\delta} \\
\Delta w^{x} \\
\Delta w^{p}
\end{array}\right)=-\left(\begin{array}{c}
J_{k}^{\mathrm{T}} H_{k}-w_{k} \\
\operatorname{diag}\left(\delta_{k}\right) w_{k}^{\delta}-\mu e \\
\operatorname{diag}\left(x_{k}\right) w_{k}^{x}-\mu e \\
\operatorname{diag}\left(p_{k}\right) w_{k}^{p}-\mu e
\end{array}\right) .
$$

In other words, I omit the second-order information of the system of equations $H(z)=0$. The second-order term $L_{k} H_{k}$ can be neglected, as $H_{k}$ is small near the solution $z_{k}$ of system (6). Most of the computational cost of an interior-point method is associated with the computation of the search direction. By exploiting the special features of the problem, I reduce the computational cost within the algorithm in terms of function evaluations and number of iterations.

In addition, some components of the next iterate $\left(z_{k+1}, w_{k+1}^{\delta}, w_{k+1}^{x}, w_{k+1}^{p}\right)$ should be forced to remain strictly positive, a requirement that is achieved by rescaling the search direction $\left(\Delta z, \Delta w^{\delta}, \Delta w^{x}, \Delta w^{p}\right)$. In particular, to ensure $\delta_{k+1}, x_{k+1}, p_{k+1}, w_{k+1}^{\delta}$, $w_{k+1}^{x}, w_{k+1}^{p}>0$, scalars $\alpha_{\delta_{k}}, \alpha_{x_{k}}, \alpha_{p_{k}}, \alpha_{w_{k}^{\delta}}, \alpha_{w_{k}^{x}}$ and $\alpha_{w_{k}^{p}}$ must be chosen such that $\delta_{k}+\alpha_{\delta_{k}} \Delta \delta>0, \quad x_{k}+\alpha_{x_{k}} \Delta x>0, \quad p_{k}+\alpha_{p_{k}} \Delta p>0, \quad w_{k}^{\delta}+\alpha_{w_{k}^{\delta}} \Delta w_{k}^{\delta}>0, \quad w_{k}^{x}+\alpha_{w_{k}^{x}} \Delta w_{k}^{x}>0$ and $w_{k}^{p}+\alpha_{w_{k}^{p}} \Delta w_{k}^{p}>0$. These parameters are called steplength parameters. Therefore, at iteration $k$,

$$
\begin{aligned}
& \alpha_{\delta_{k}}=\min \left\{1, \min \left\{-\frac{\delta_{k i}}{\Delta \delta_{i}} \text { s.t. } \Delta \delta_{i}<0\right\}\right\}, \\
& \alpha_{x_{k}}=\min \left\{1, \min \left\{-\frac{x_{k i}}{\Delta x_{i}} \text { s.t. } \Delta x_{i}<0\right\}\right\}, \\
& \alpha_{p_{k}}=\min \left\{1, \min \left\{-\frac{p_{k i}}{\Delta p_{i}} \text { s.t. } \Delta p_{i}<0\right\}\right\}, \\
& \alpha_{w_{k}^{\delta}}=\min \left\{1, \min \left\{-\frac{w_{k i}^{\delta}}{\Delta w_{k i}^{\delta}} \text { s.t. } \Delta w_{k i}^{\delta}<0\right\}\right\}, \\
& \alpha_{w_{k}^{x}}=\min \left\{1, \min \left\{-\frac{w_{k i}^{x}}{\Delta w_{k i}^{x}} \text { s.t. } \Delta w_{k i}^{x}<0\right\}\right\},
\end{aligned}
$$

and

$$
\alpha_{w_{k}^{p}}=\min \left\{1, \min \left\{-\frac{w_{k i}^{p}}{\Delta w_{k i}^{p}} \text { s.t. } \Delta w_{k i}^{p}<0\right\}\right\},
$$

where $\left(\delta_{k i}, x_{k i}, p_{k i}, w_{k i}^{\delta}, w_{k i}^{x}, w_{k i}^{p}\right)$ and $\left(\Delta \delta_{i}, \Delta x_{i}, \Delta p_{i}, \Delta w_{k i}^{\delta}, \Delta w_{k i}^{x}, \Delta w_{k i}^{p}\right)$ are the $i$ th component of vectors $\left(\delta_{k}, x_{k}, p_{k}, w_{k}^{\delta}, w_{k}^{x}, w_{k}^{p}\right)$ and $\left(\Delta \delta, \Delta x, \Delta p, \Delta w^{\delta}, \Delta w^{x}, \Delta w^{p}\right)$.

An additional condition on $\alpha$ is required to ensure global convergence of $\left\{z_{k}\right\}$; namely, the scalar $\alpha_{z_{k}}$ should be chosen such that the objective function $\frac{1}{2}\|H(z)\|_{2}^{2}-$ $\mu(\log \delta+\log x+\log p)$ decreases sufficiently in each iteration $z_{k}$ (Armijo's rule) and 
the choice $\alpha_{z_{k}}$ is not too far from a minimizer of the objective function (Goldstein's rule). If these requirements are not satisfied, $\alpha_{z_{k}}$ should be modified. Because these criteria help us to find an appropriate step length $\alpha_{z_{k}}$, they are called line-search methods. This procedure relies on a univariate function called merit function $m(\alpha)$, to measure the progress of the algorithm. A suitable merit function for the algorithm is

$$
m(\alpha)=\frac{1}{2}\|H(z+\alpha \Delta z)\|_{2}^{2}-\mu(\log (\delta+\alpha \Delta \delta)+\log (x+\alpha \Delta x)+\log (p+\alpha \Delta p)) .
$$

(Other examples of merit functions can be found in Nocedal and Wright, 1999). Then $\alpha_{z_{k}}$ results in a sufficient decrease if

$$
\begin{aligned}
& m\left(\alpha_{z_{k}}\right) \leqslant m(0)+\rho \alpha_{z_{k}} \nabla m(0)^{\mathrm{T}} \Delta z, \\
& \left|\nabla m\left(\alpha_{z_{k}}\right)^{\mathrm{T}} \Delta z\right| \leqslant \eta\left|\nabla m(0)^{\mathrm{T}} \Delta z\right|,
\end{aligned}
$$

where $0<\rho<\eta<1$. Otherwise $\alpha_{z_{k}}$ should be reduced until conditions (9) and (10) are satisfied. In particular, when $\alpha_{z_{k}}$ does not satisfy (9) and (10), then I consider an update of the steplength as $\alpha_{z_{k}} / 2$.

The complete iteration of the algorithm requires an update of the barrier parameter $\mu$. This update should be carefully defined to obtain a rapidly convergent algorithm. The choice of $\mu$ is based on the satisfaction of the complementarity conditions $w=\mu \Sigma^{1} e$. Then the new value of $\mu$ at the $k$ th iteration is

$$
\mu_{k+1}=\gamma \frac{z_{k}^{\mathrm{T}} w_{k}}{n}=\gamma \frac{\delta_{k}^{\mathrm{T}} w_{k}^{\delta}+x_{k}^{\mathrm{T}} w_{k}^{x}+p_{k}^{\mathrm{T}} w_{k}^{p}}{n},
$$

where $0 \leqslant \gamma<1$ and $n$ is the number of simple bounds (i.e. the dimension of vector $(\delta, x, p))$. This definition ensures that $\mu \rightarrow 0$ if Problem (5) has a solution. When I choose $\gamma$ close to 0 , I am requiring a rapid convergence of $\mu$ to 0 . The choice of the updating parameter $\gamma$ may significantly affect the efficiency of the overall method.

The algorithm terminates when the following stopping criteria are satisfied:

$$
\left\|\left(\begin{array}{c}
J(z)^{\mathrm{T}} H(z)-w=0 \\
\operatorname{diag}(\delta) w^{\delta}-\mu e=0 \\
\operatorname{diag}(x) w^{x}-\mu e=0 \\
\operatorname{diag}(p) w^{p}-\mu e=0
\end{array}\right)\right\|_{2}^{2} \leqslant \varepsilon_{\mathrm{SIZE}},
$$

The stopping criterion (12), hereafter called the first stopping criterion, is related to the fulfillment of the first-order KKT conditions for Problem (5) and guarantees the boundedness of the variables. The stopping criterion (13), which I call the second stopping criterion, guarantees satisfaction of the optimality conditions for the existence of equilibria under convexity assumptions. Therefore, if the algorithm converges, it converges to an equilibrium of the economy. 


\subsection{The algorithm: general formulation}

In the preceding description of the algorithm, I have considered simple lowerbounds in order to simplify the exposition. The generalization of this algorithm to problems such that $H(z)=0$ where $z$ lie between the given bounds $l>-\infty$ and $u<\infty$ (i.e. $l \leqslant z \leqslant u$ ) is straightforward. First, I rewrite the problem as $H(z)=0$ with $z-l \geqslant 0$ and $u-z \geqslant 0$, and then the finite bounds will be included in the objective function via logarithmic barrier terms. Therefore, I consider two auxiliary vectors of variables $w^{1}$ and $w^{2}$, defined by the nonlinear transformation $w^{1}=\mu(z-l)^{1}$ and $w^{2}=\mu(u-z)^{1}$. Assuming that $z \in \mathbb{R}^{N}$, there $2 N$ bounds of the type $z-l \geqslant 0$ and $u-z \geqslant 0$, and therefore, each auxiliary vector $w^{1}$ and $w^{2}$ has $N$ components. Let $L, U, Z, W^{1}, W^{2}$ be diagonal matrices, the diagonal entries of which are the components of vector $l, u, z, w^{1}, w^{2}$.

A summary of the proposed interior-point algorithm is:

Step 1 . Select the parameters $\rho \in(0,1), \theta \in(0,1), \gamma \in[0,1)$, the tolerance $\varepsilon_{\text {SIZE }}$, and the final stop tolerance $\varepsilon_{\mathrm{TOL}}$. Initialize variables $\left(z, w^{1}, w^{2}\right)$ and set the initial value of the barrier parameter $\mu$ as

$$
\mu=\gamma \frac{(z-l)^{\mathrm{T}} w^{1}+(u-z)^{\mathrm{T}} w^{2}}{2 N} .
$$

Step 2. Evaluate the objective function and its derivatives at $\left(z, w^{1}, w^{2}\right)$. Repeat until

$$
\left\|\left(\begin{array}{c}
J^{\mathrm{T}} H-w^{1}+w^{2} \\
(Z-L) w^{1}-\mu e \\
(U-Z) w^{2}-\mu e
\end{array}\right)\right\| \leqslant \varepsilon_{\mathrm{TOL}}
$$

and (13) are satisfied:

Step 2.1. Compute a Newton search direction:

$$
\left(\begin{array}{ccc}
J^{\mathrm{T}} J & -I & I \\
W^{1} & (Z-L) & 0 \\
-W^{2} & 0 & (U-Z)
\end{array}\right)\left(\begin{array}{c}
\Delta z \\
\Delta w^{1} \\
\Delta w^{2}
\end{array}\right)=-\left(\begin{array}{c}
J^{\mathrm{T}} H-w^{1}+w^{2} \\
(Z-L) w^{1}-\mu e \\
(U-Z) w^{2}-\mu e
\end{array}\right) .
$$

Step 2.2. Compute scalars $\alpha_{z}, \alpha_{w^{1}}$ and $\alpha_{w^{2}}$ such that:

$$
\begin{aligned}
& \alpha_{z}=\min \left\{1, \min \left\{-\frac{\left(z_{i}-l_{i}\right)}{\Delta z_{i}} \text { s.t. } \Delta z_{i}<0\right\}, \min \left\{-\frac{\left(u_{i}-z_{i}\right)}{\Delta z_{i}} \text { s.t. } \Delta z_{i}<0\right\}\right\}, \\
& \alpha_{w^{1}}=\min \left\{1, \min \left\{-\frac{w_{i}^{1}}{\Delta w_{i}^{1}} \text { s.t. } \Delta w_{i}^{1}<0\right\}\right\}, \\
& \alpha_{w^{2}}=\min \left\{1, \min \left\{-\frac{w_{i}^{2}}{\Delta w_{i}^{2}} \text { s.t. } \Delta w_{i}^{2}<0\right\}\right\},
\end{aligned}
$$

and update $\alpha_{z}$ until (9) and 10) are satisfied. 
Step 2.3. Update variables as $z+\alpha_{z} \Delta z$ and $w+\alpha_{w} \Delta w$.

Step 2.4. Update parameter $\mu$ as described in (14).

Some of the entries in $l$ and $u$ can be equal to $-\infty$ and $\infty$. If the $i$ th component of $l_{i}$ is equal to $-\infty$ and the $i$ th component of $u_{i}$ is equal to $\infty$, the $i$ th component of variable $z$ is unconstrained, and the $i$ th component of the auxiliary variables $w^{1}$ and $w^{2}$ are zero. This is the case presented in the beginning of Section 3. In case that the $i$ th component of $l_{i}$ is equal to $-\infty$ but the $i$ th component of $u_{i}$ is less than $\infty$, the $i$ th component of the auxiliary variable $w^{1}$ should be greater than zero and the $i$ th component of the auxiliary variable $w^{2}$ should be zero. The same argument can be applied to any variable in the model.

There are many different types of interior-point algorithms with certain common mathematical themes having to do with the logarithmic barrier function. The distinguishing feature of the algorithm is the use of a Gauss-Newton approximation of the search direction. In the Appendix I analyze the convergence properties of this variant and prove that, under mild conditions, the algorithm globally converges to KKT points (i.e. solution points of the system of equations given by (2)-(4)). Also I prove that the algorithm converges quadratically, which broadly means that the number of correct figures in $z_{k}$ eventually doubles at each step.

\subsection{Practical implementation issues}

The algorithm described in the preceding section includes certain parameters and conditions that have not yet been completely specified. In the following paragraphs I indicate how these implementation issues were treated.

\subsubsection{Choosing the initial point}

The algorithm performs better if the starting point $\left(z_{0}, w_{0}\right)$ meets the bound constraints. For simplicity, the algorithm sets the auxiliary variables $w_{0}$ and $\delta_{0}, \theta_{0}, p_{0}, q_{0}$ equal to a vector of ones and $x_{0}$ equal to the initial endowment.

\subsubsection{Choosing the parameters}

In the implementation, a current iterate is considered optimal when $\varepsilon_{\mathrm{TOL}}=10^{14}$ and $\varepsilon_{\text {SIZE }}=10^{10}$. The Armijo and Goldstein parameters are $\rho=0.0001$ and $\eta=0.9$. The choice of the parameter $\mu$ is based on the satisfaction of the complementarity conditions and depends on the parameter $\gamma$. Typically, $\gamma=0.1$.

\subsubsection{Computing the search direction}

The computationally most expensive part of an interior-point algorithm is the computation of the Newton search direction, because this calculation involves the solution of a potentially large system of linear equations. It is important to note that the matrix

$$
\left(\begin{array}{cc}
J_{k}^{\mathrm{T}} J_{k} & -I \\
W_{k} & Z_{k}
\end{array}\right)
$$


is sparse - that it contains a significant number of zero-valued elements. Note that matrices $I, W_{k}$, and $Z_{k}$ are diagonal and, furthermore, that $J_{k}$ is of the form

$$
J_{k}=\left(\begin{array}{ccccc}
\nabla_{x} u & \nabla_{x}^{2} u & 0 & -I & 0 \\
0 & P & V & X & U \\
0 & 0 & 0 & \Phi A & 0 \\
0 & I & 0 & 0 & 0 \\
0 & 0 & I & 0 & 0
\end{array}\right),
$$

where $\nabla_{x} u$ and $\nabla_{x}^{2} u$ are diagonal matrices because the vector has the form $\left(u_{1}\left(x_{10}, \ldots, x_{1 S}\right), \ldots, u_{I}\left(x_{I 0}, \ldots, x_{I S}\right)\right) ; P$ is the diagonal matrix of the prices vector $\left(p_{0}, p_{1}, \ldots, p_{S}\right) ; X$ is the diagonal matrix of the consumption allocations for all consumers $\left(x_{10}, \ldots, x_{1 S}, \ldots, x_{i 0}, \ldots, x_{i S}, \ldots, x_{I 0}, \ldots, x_{I S}\right) ; \Phi$ is the diagonal matrix of the auxiliary variables $\left(\delta_{10}, \ldots, \delta_{1 S}, \ldots, \delta_{i 0}, \ldots, \delta_{i S}, \ldots, \delta_{I 0}, \ldots, \delta_{I S}\right) ; V$ is the full matrix of returns; and $U$ is an auxiliary matrix, respectively, defined as

$$
V=\left(\begin{array}{c}
q \\
-A
\end{array}\right), \quad U=\left(\begin{array}{c}
e_{1 \times C} \\
0_{S \times C}
\end{array}\right) .
$$

This sparsity can and should be exploited to improve the computational efficiency. In the computational results reported in the next section, I exploit the sparsity properties of the full matrix (15), which reduces computation time by eliminating operations on zero elements.

\section{Numerical examples}

The algorithm has been implemented in MATLAB 6.5 on an Intel Centrino Pentium M $1.6 \mathrm{GHz}$ with machine precision $10^{16}$. The first example is intended to show how the algorithm is set up and to compute a GEI equilibrium.

Example 2 (Two-period exchange economy. DeMarzo and Eaves, 1996). Consider a two-period exchange economy with three consumers, three states in the second period, two assets, and two goods. Each consumer $i$ has a utility function of the form $u_{i}(x)=\sum_{s=0}^{3} \pi_{s}\left(B-x_{s 1}^{\alpha_{i}} x_{s 2}^{1} \alpha_{i}\right)$, with parameters $B=57, \pi=\left(1, \frac{1}{3}, \frac{1}{3}, \frac{1}{3}\right), \alpha_{1}=\alpha_{2}=\frac{3}{4}$ and $\alpha_{3}=\frac{1}{4}$. The agent's endowments are $w_{1}=w_{2}=(10,10 ; 25,20 ; 20,20 ; 15,20)^{\mathrm{T}}$ and $w_{3}=(20,20 ; 5,10 ; 10,10 ; 15,20)^{\mathrm{T}}$. The asset matrix $A$ is given by

$$
A^{\mathrm{T}}=\left[\begin{array}{cccccc}
1 & 0 & 1 & 0 & 1 & 0 \\
2 & -1 & 1 & 0 & 2 & -1
\end{array}\right]
$$

For this example, $H(\cdot)=0$ is a system of 52 equations withh 52 variables (the auxiliary variables $\delta^{*} \in \mathbb{R}_{++}^{12}$ and the equilibrium $\left.\left(x^{*}, \theta^{*}, p^{*}, q^{*}\right) \in \mathbb{R}^{40}\right)$. Taking as an initial point $\delta_{0}=e, x_{10}=w_{1}, x_{20}=w_{2}, x_{20}=w_{3}$ and $\theta_{0}, p_{0}, q_{0}=e$, where $e$ is a vector of ones, the algorithm converges in $0.56 \mathrm{~s}$ (20 iterations) with an error of $\varepsilon_{\text {SIZE }}=10^{14}$. Table 1 shows the iterates of the portfolio allocations for each iteration until convergence. $\theta(i, c)$ denotes the portfolio decision of the asset $c$ by agent $i$. 
Table 1

Iterates of the portfolio allocations approaching convergence in Example 2

\begin{tabular}{lrrrrrr}
\hline Iterations & $\theta(1,1)$ & $\theta(1,2)$ & $\theta(2,1)$ & $\theta(2,2)$ & $\theta(3,1)$ & $\theta(3,2)$ \\
\hline 1 & 1.0000 & 1.0000 & 1.0000 & 1.0000 & 1.0000 & 1.0000 \\
2 & 7.5882 & 7.4157 & 13.6431 & 13.4706 & 20.3205 & 21.7970 \\
3 & 5.3402 & 3.1748 & 11.1594 & 7.7269 & 16.4392 & 10.9132 \\
4 & 1.6257 & 4.5227 & 10.2507 & 12.8887 & 11.8786 & 17.4080 \\
5 & 4.5801 & 6.4406 & 8.3884 & 9.3628 & 12.9683 & 15.8037 \\
6 & 1.6009 & 5.3987 & 2.5987 & 6.2855 & 4.1996 & 11.6842 \\
7 & 1.2995 & 3.9109 & 1.0984 & 4.0964 & 2.3979 & 8.0074 \\
8 & 0.7307 & 4.3251 & 0.7121 & 4.3375 & 1.4428 & 8.6626 \\
9 & 0.6793 & 4.4071 & 0.6604 & 4.4248 & 1.3397 & 8.8319 \\
10 & 0.6463 & 4.4258 & 0.6464 & 4.4253 & 1.2927 & 8.8512 \\
11 & 0.6359 & 4.4387 & 0.6342 & 4.4404 & 1.2700 & 8.8791 \\
12 & 0.6355 & 4.4379 & 0.6356 & 4.4378 & 1.2711 & 8.8757 \\
13 & 0.6340 & 4.4397 & 0.6338 & 4.4398 & 1.2678 & 8.8795 \\
14 & 0.6342 & 4.4393 & 0.6342 & 4.4393 & 1.2685 & 8.8786 \\
15 & 0.6340 & 4.4395 & 0.6340 & 4.4396 & 1.2680 & 8.8791 \\
16 & 0.6341 & 4.4395 & 0.6341 & 4.4395 & 1.2681 & 8.8790 \\
17 & 0.6340 & 4.4395 & 0.6340 & 4.4395 & 1.2681 & 8.8790 \\
18 & 0.6340 & 4.4395 & 0.6340 & 4.4395 & 1.2681 & 8.8790 \\
19 & 0.6340 & 4.4395 & 0.6340 & 4.4395 & 1.2681 & 8.8790 \\
20 & 0.6340 & 4.4395 & 0.6340 & 4.4395 & 1.2681 & 8.8790 \\
\hline
\end{tabular}

Table 2 shows the values of the first and second stopping criteria and the computation time for each iteration until convergence, which reveal the good performance of the algorithm, even though it started from a poor initial point.

The following computations are intended to demonstrate how the algorithm behaves in large-scale markets.

Example 3 (Large-scale computations). I consider four variations of the two-period exchange economy described in DeMarzo and Eaves (1996). All the models consider a two-period exchange economy with two goods, two assets with the asset matrix $A$ given in Example 1, and three states in the second period. Endowments and constants of the utility function were randomly generated using: $w_{i} \sim \mathrm{U}[0.75,1.25]$ and $\alpha_{i} \sim \mathrm{U}[0,1]$ for each consumer $i$. However, Model 1 considers three agents, Model 2 has 15 agents, Model 3 consists of 30 agents, and Model 4 has 60 agents. Table 3 shows the number of variables and equations for each model and the number of iterations and running times until convergence with an error of $\varepsilon_{\text {SIZE }}=10^{14}$. For all models, the algorithm converges to an equilibrium in a moderate number of iterations, which illustrates its rapid convergence. The scale of the problem only affects the cost of computation, mainly because of the cost of function evaluations.

The fourth example illustrates the behavior of the algorithm if the economy has no equilibrium. 
Table 2

Iterates of the first and second stopping criteria, the penalty parameter $\mu$, and the cumulative $\mathrm{CPU}$ time (seconds) until convergence

\begin{tabular}{lrrll}
\hline Iterations & 1st stopping criterion & 2nd stopping criterion & $\mu$ & CPU time \\
\hline 0 & 8915.4541 & 8500.9862 & 0.9091 & 0.06 \\
1 & 3477.7741 & 98373.5254 & 0.0941 & 0.1 \\
2 & 1488.1517 & 71632.9528 & 0.0209 & 0.11 \\
3 & 238.2734 & 112039.2402 & 0.0086 & 0.12 \\
4 & 2.4820 & 9962.1706 & 0.0019 & 0.13 \\
5 & 0.1586 & 2032.0153 & 0.0004 & 0.15 \\
6 & 0.1300 & 847.0930 & 0.0001 & 0.16 \\
7 & 0.0209 & 340.7255 & 0.0000 & 0.17 \\
8 & 0.0069 & 60.4907 & 0.0000 & 0.18 \\
9 & 0.0012 & 12.7272 & 0.0000 & 0.19 \\
10 & 0.0004 & 2.4646 & 0.0000 & 0.2 \\
11 & 0.0001 & 0.6478 & 0.0000 & 0.21 \\
12 & 0.0000 & 0.2791 & 0.0000 & 0.23 \\
13 & 0.0000 & 0.1302 & 0.0000 & 0.24 \\
14 & 0.0000 & 0.0762 & 0.0000 & 0.25 \\
15 & 0.0000 & 0.0409 & 0.0000 & 0.26 \\
16 & 0.0000 & 0.0272 & 0.0000 & 0.27 \\
17 & 0.0000 & 0.0113 & 0.0000 & 0.29 \\
18 & 0.0000 & 0.0100 & 0.0000 & 0.3 \\
19 & 0.0000 & 0.0003 & 0.0000 & 0.31 \\
20 & 0.0000 & 0.0000 & 0.0000 & 0.33 \\
\hline
\end{tabular}

Table 3

Number of iterations and running times for four different models

\begin{tabular}{lclr}
\hline & Number variables/equations & Number of iterations & CPU (seconds) \\
\hline Model 1: 3 agents & 52 & 22 & 0.51 \\
Model 2: 15 agents & 260 & 26 & 4.09 \\
Model 3: 30 agents & 520 & 29 & 20.71 \\
Model 4: 60 agents & 1040 & 32 & 216.89 \\
\hline
\end{tabular}

Example 4 (One-period exchange economy with no equilibria). Suppose there is no first period. Consider a one-period exchange economy with two consumers, two states, two assets, and two goods. Each consumer $i$ has a utility function of the form $U_{i}(x)=u_{i}\left(x_{1}\right)+u_{i}\left(x_{2}\right)$, with

$$
u_{i}\left(x_{1}\right)=\frac{1}{3} \log \left(x_{s 1}\right)+\frac{2}{3} \log \left(x_{s 2}\right), \quad u_{i}\left(x_{2}\right)=\frac{2}{3} \log \left(x_{s 1}\right)+\frac{1}{3} \log \left(x_{s 2}\right) .
$$

The agent's endowments are $w_{1}=(1-\varpi, 1-\varpi, \varpi, \varpi)^{\mathrm{T}}$ and $w_{2}=(\varpi, \varpi, 1-\varpi, 1-\varpi)^{\mathrm{T}}$ for some positive $\varpi<\frac{1}{2}$. For this example, $H\left(\delta^{*}, x^{*}, \theta^{*}, p^{*}, q^{*}\right)=0$ is a system of 20 equations with 20 variables. I assume that there are bounds only on the auxiliary variables $\delta^{*} \in \mathbb{R}_{++}^{4}$, i.e. $l \leqslant \delta^{*}$ with $l=(0,0,0,0)$. 
This economy has no equilibrium (see Hart, 1975) and the algorithm does not converge. The collinearity of the return matrix makes system (7) incompatible, and consequently the algorithm indicates that an unbounded search direction in the portfolios iterates should be taken from the initial point (the computed search direction is infinite). In other words, the algorithm fails to converge.

\section{Summary and conclusions}

In this paper I describe an efficient algorithm for the computation of equilibria in general equilibrium models with incomplete asset markets. The procedure is based on an interior-point method to define the search direction for the new iterates. Particular care has been taken to reduce the computational cost, avoiding the use of second-order information for the more complicated elements of the problem. The algorithm has proven to be globally convergent and the local convergence rate is quadratic.

Given its practicability and efficiency, this algorithm seems to be a interesting alternative for computing equilibria when the existing homotopy continuation approaches are difficult to apply.

\section{Acknowledgements}

The author wishes to thank an anonymous referee for numerous and helpful comments. This research has been supported by the European Commission through project HPMF-CT-2000-00781.

\section{Appendix A}

\section{A.1. Convergence properties of the algorithm}

To prove convergence properties of the proposed algorithm, we must ensure a sufficient descent on the merit function in every iteration, and this function should be bounded below. For the sake of a clearer presentation, I consider the problem: $H\left(z^{*}\right)=0$, where $z^{*} \in \mathbb{R}_{+}^{N}$, and let $w$ be an auxiliary variable, so that $w=\mu Z{ }^{1} e$ where $Z=\operatorname{diag}(z)$. Then, let us denote

$$
\mathscr{F}_{\mu}\left(z_{k}, w_{k}\right)=\left(\begin{array}{c}
J_{k}^{\mathrm{T}} H_{k}-w_{k} \\
Z_{k} W_{k}-\mu_{k} e
\end{array}\right), \quad \mathscr{H}_{\mu}\left(z_{k}, w_{k}\right)=\left(\begin{array}{cc}
J_{k}^{\mathrm{T}} J_{k} & -I \\
W_{k} & Z_{k}
\end{array}\right) .
$$

The convergence of the algorithm is obtained under the following conditions:

A.1. The second derivatives of $H$ are Lipschitz continuous on the region defined by the bounds.

A.2. Strict complementarity holds at all first-order KKT points (i.e. $w_{i}^{*}>0$ for $i \in$ $\left\{i \mid z_{i}^{*}=0\right\}$ and vice versa).

A.3. The matrix $J$ has full rank at all first-order KKT points. 
A.4. For a positive constant $M$, and any vector $v \in \mathbb{R}^{N}$

$$
\frac{1}{M}\|v\|^{2} \leqslant v^{\mathrm{T}} J^{\mathrm{T}} J v \leqslant M\|v\|^{2} .
$$

The basic GEI model describes an economy with agents represented by smooth, strictly monotone and strictly convex preferences. The smoothness property implies Condition A.1. Conditions A.2 and A.3 ensure that the matrix $\mathscr{H}_{\mu}\left(z_{k}, w_{k}\right)$ is nonsingular (assuming Condition A.1, both statements are equivalent) and the inverse of $\mathscr{H}_{\mu}\left(z_{k}, w_{k}\right)$ is uniformly bounded near the solution. Condition A.3 is trivially satisfied if $A_{s}$ has full rank and strict complementarity holds. Condition A.2 limits the applicability of the algorithm, as there could be economies with equilibria which do not satisfy the strict complementarity assumption. In these cases, we can consider other interior-point approaches, for example, Heinkenschloss et al. (1999). Condition A.4 holds under strictly convex preferences assumption. Conditions A.3 and A.4 ensure the positive definiteness of $J^{\mathrm{T}} J$, which guarantees that the search direction is always well-defined.

I start by stating the boundedness of dual variables $\left\{w_{k}\right\}$.

Lemma 5. For a fixed $\mu$, the lower bounds and the upper bounds of the box constraints in the dual step-size rule are bounded away from zero and bounded from above, if the corresponding components of $z_{k}$ are also bounded above and away from zero.

Proof. See Yamashita (1998).

As a consequence, the elements of $Z_{k}{ }^{1} W_{k}$ are bounded above and non-zero and, by Assumption A.4, there exists a positive constant $N$ such that $(1 / N)\|v\|^{2} \leqslant v^{\mathrm{T}}\left(J_{k}^{\mathrm{T}} J_{k}+Z_{k}^{1} W_{k}\right) v \leqslant N\|v\|^{2}$.

I now show that $\Delta z$ is a descent direction for the merit function $m(\alpha)$.

Theorem 6. For a fixed $\mu, \Delta z$ is a descent direction for the merit function $m(\alpha)$, i.e. $\nabla m(0)^{\mathrm{T}} \Delta z \leqslant 0$.

Proof. Note that the direction $\Delta z$ is given as

$$
\left(J_{k}^{\mathrm{T}} J_{k}+Z_{k}{ }^{1} W_{k}\right) \Delta z=-\left(J_{k}^{\mathrm{T}} H_{k}-\mu Z_{k}{ }^{1} e\right) .
$$

Then

$$
\begin{aligned}
\Delta z^{\mathrm{T}} \nabla m(0) & =\Delta z^{\mathrm{T}}\left(J_{k}^{\mathrm{T}} H_{k}-\mu Z_{k}{ }^{1} e\right) \\
& =-\Delta z^{\mathrm{T}}\left(J_{k}^{\mathrm{T}} J_{k}+Z_{k}{ }^{1} W_{k}\right) \Delta z \leqslant 0 .
\end{aligned}
$$

By definition, the step size $\alpha$ is always bounded. I next prove that $\alpha$ is also non-zero.

Lemma 7. The step size $\alpha$ is always bounded and non-zero.

Proof. Assume that $\alpha$ is reduced infinitely. This means that the descent condition does not hold; therefore

$$
m(0)-m(\alpha) \leqslant-\rho \alpha \nabla m(0)^{\mathrm{T}} \Delta z^{\mathrm{T}}, \quad \text { for all } \alpha .
$$


From the Taylor expansion, I have $m(\alpha)-m(0)=\alpha \nabla m(0)^{\mathrm{T}} \Delta z+\mathrm{o}(\alpha)$. Then for a small enough $\alpha$, using Theorem 6 ,

$$
m(\alpha)-m(0)-\rho \alpha \nabla m(0)^{\mathrm{T}} \Delta z^{\mathrm{T}}=(1-\rho) \alpha \nabla m(0)^{\mathrm{T}} \Delta z^{\mathrm{T}} \leqslant 0,
$$

which contradicts (16).

The next two results prove the global convergence of the algorithm for a fixed $\mu$.

Lemma 8. For a fixed $\mu$, assume also that at any initial point $z_{0}$, the set $\{z$ : $\left.m\left(z_{k} ; \mu\right) \leqslant m\left(z_{0} ; \mu\right)\right\}$ is bounded. Then $\lim _{k \rightarrow \infty}\|\Delta z\|=0$.

Proof. Assume $\lim _{k \rightarrow \infty}\|\Delta z\| \geqslant \varepsilon>0$. Using the descent lemma (see Bertsekas, 1995, p. 553)

$$
m\left(z_{k+1} ; \mu\right)-m\left(z_{k} ; \mu\right) \leqslant \alpha \nabla m\left(z_{k}\right)^{\mathrm{T}} \Delta z+\frac{1}{2} K \alpha^{2}\|\Delta z\|^{2} \leqslant \frac{1}{2} K \alpha^{2}\|\Delta z\|^{2}
$$

for some positive constant $K$, as $m$ is a Lipschitz continuous function and $\Delta z$ is a descent direction of the merit function $m$, using Theorem 6 .

By hypothesis, the sequence $\left\{m\left(z_{k} ; \mu\right)\right\}$ converges and $\lim _{k \rightarrow \infty}\left(m\left(z_{k} ; \mu\right)\right.$ $\left.-m\left(z_{k+1} ; \mu\right)\right)=0$, thus

$$
m\left(z_{k} ; \mu\right)-m\left(z_{k+1} ; \mu\right) \geqslant-\frac{1}{2} K L \alpha^{2}\|\Delta z\|^{2} \rightarrow 0,
$$

which implies $\|\Delta z\| \rightarrow 0$, using Lemma 7.

Theorem 9. For a fixed $\mu$, the algorithm terminates at a point, satisfying the perturbed optimality conditions (6).

Proof. As $\Delta w=-\left(W_{k}-\mu Z_{k}{ }^{1} e+Z_{k}{ }^{1} W_{k} \Delta z\right)$ and $\lim _{k \rightarrow \infty}\|\Delta z\|=0$, by Lemma 8 , the following holds:

$$
\lim _{k \rightarrow \infty}\left\|w_{k}+\Delta w-\mu Z_{k}^{1} e\right\|=0 .
$$

Therefore, there exists a vector $w^{*}$ such that $\lim _{k \rightarrow \infty}\left\|w_{k}+\Delta w\right\|=\mu Z_{*}{ }^{1} e$. Furthermore, the first equation of system (6) satisfies

$$
\left(J_{k}^{\mathrm{T}} J_{k}+Z_{k}{ }^{1} W_{k}\right) \Delta z=-\left(J_{k}^{\mathrm{T}} H_{k}-\mu Z_{k}{ }^{1} e\right)
$$

and letting $k \rightarrow \infty$,

$$
\lim _{k \rightarrow \infty}\left\|J_{k}^{\mathrm{T}} H_{k}-\mu Z_{k}{ }^{1} e\right\|=0
$$

i.e. there exists a $z^{*}$ such that $J_{*}^{\mathrm{T}} H_{*}-\mu Z_{*}{ }^{1} e=0$. Then $\left(z^{*}, w^{*}\right)$ is a solution of the perturbed optimality conditions (6).

The convergence of the algorithm can be proven using the next theorem.

Theorem 10. Suppose that Assumptions A.1-A.4 hold. The limit of sequence $\left\{\left(z_{k}, w_{k}\right)\right\}$ exists and satisfies the optimality conditions of Problem (5).

Proof. Note that the definition of $\mu_{k}$ ensures that $\left\{\mu_{k}\right\}$ is positive and monotonically decreasing with $\mu \rightarrow 0$. Thus, it follows from Theorems 9 and 10 that the algorithm terminates at a point $\left(z_{k}, w_{k}\right)$, satisfying the optimality conditions of Problem (5). 
Using analogous arguments to those discussed in Akrotirianakis and Rustem (2000), it is satisfied $\lim _{k \rightarrow \infty}\left\|\mathscr{F}_{\mu}\left(z_{k}, w_{k}\right)\right\|_{2}=0$.

Next I prove the $Q$-quadratic convergence of the algorithm. First, I present an auxiliary result.

Lemma 11. Suppose that Assumptions A.1-A.4 hold. Then

$$
\left\|\Lambda_{k}-I\right\| \leqslant \mathrm{O}\left(\left\|\mathscr{F}_{\mu}\left(z_{k}, w_{k}\right)\right\|\right)+\mathrm{O}\left(\mu_{k}\right),
$$

where $\Lambda_{k}=\operatorname{diag}\left(\alpha_{k}\right)$.

Proof. See Yamashita and Yabe (1996).

Using this lemma, I establish the quadratic convergence of the algorithm.

Theorem 12. Suppose that Assumptions A.1-A.4 hold and that the sequence $\left\{\left(z_{k}, w_{k}\right)\right\}$ generated by the proposed algorithm converges to the solution $\left(z^{*}, w^{*}\right)$. Assume that $\mu_{k}=\mathrm{O}\left(\left\|\mathscr{F}_{\mu}\left(z_{k}, w_{k}\right)\right\|\right)$. Then there exists $\varepsilon>0$ such that for all $\left(z_{0}, w_{0}\right) \in B\left(\left(z^{*}, w^{*}\right), \varepsilon\right)$, the sequence $\left\{\left(z_{k}, w_{k}\right)\right\}$ is well defined and converges to $\left(z^{*}, w^{*}\right) Q$-quadratically.

Proof. Denote $h_{k}=\left(z_{k}, w_{k}\right)$ and $h^{*}=\left(z^{*}, w^{*}\right)$. Since $h_{0} \in B\left(h^{*}, \varepsilon\right),\left\|h_{0}-h^{*}\right\|<\varepsilon$. Assume $\left\|h_{k}-h^{*}\right\|<\varepsilon$, then

$$
\begin{aligned}
h_{k+1}-h^{*}= & h_{k}+\Lambda_{k}(\Delta z, \Delta w)-h^{*}=h_{k}-\Lambda_{k} \mathscr{H}_{\mu}\left(h_{k}\right){ }^{1} \mathscr{F}_{\mu}\left(h_{k}\right)-h^{*} \\
= & \mathscr{H}_{\mu}\left(z_{k}, w_{k}\right){ }^{1}\left[\Lambda_{k} \mathscr{F}_{\mu}\left(h^{*}\right)-\Lambda_{k} \mathscr{F}_{\mu}\left(h_{k}\right)+\mathscr{H}_{\mu}\left(z_{k}, w_{k}\right)\left(h_{k}-h^{*}\right)\right] \\
= & \Lambda_{k} \mathscr{H}_{\mu}\left(z_{k}, w_{k}\right){ }^{1}\left[\mathscr{F}_{\mu}\left(h^{*}\right)-\mathscr{F}_{\mu}\left(h_{k}\right)+\mathscr{H}_{\mu}\left(z_{k}, w_{k}\right)\left(h_{k}-h^{*}\right)\right] \\
& -\left(\Lambda_{k}-1\right)\left(h_{k}-h^{*}\right) .
\end{aligned}
$$

Taking Euclidean norms, I have

$$
\begin{aligned}
\left\|h_{k+1}-h^{*}\right\| & \leqslant\left\|\mathscr{H}_{\mu}\left(z_{k}, w_{k}\right){ }^{1}\right\| \mathrm{O}\left(\left\|h_{k}-h^{*}\right\|^{2}\right)+\left\|\Lambda_{k}-1\right\|\left\|h_{k}-h^{*}\right\| \\
& \leqslant \mathrm{O}\left(\left\|h_{k}-h^{*}\right\|^{2}\right)+\left[\mathrm{O}\left(\left\|\mathscr{F}_{\mu}\left(h_{k}\right)\right\|\right)+\mathrm{O}\left(\mu_{k}\right)\right]\left\|h_{k}-h^{*}\right\| .
\end{aligned}
$$

From Assumption A.1, $H$ is Lipschitz continuous, and therefore there exists a constant $N>0$ such that, for all $\left(z_{k}, w_{k}\right) \in B\left(\left(z^{*}, w^{*}\right), \varepsilon\right)$,

$$
\left\|\mathscr{F}_{\mu}\left(h_{k}\right)\right\|=\left\|\mathscr{F}_{\mu}\left(h_{k}\right)-\mathscr{F}_{\mu}\left(h^{*}\right)\right\| \leqslant N\left\|h_{k}-h^{*}\right\| .
$$

Then there exists a constant $\xi>0$, such that $\left\|h_{k+1}-h^{*}\right\| \leqslant \xi\left\|h_{k}-h^{*}\right\|^{2}$. Hence the sequence $\left\{\left(z_{k}, w_{k}\right)\right\}$ converges Q-quadratically to $\left(z^{*}, w^{*}\right)$.

\section{References}

Akrotirianakis, I., Rustem, B., 2000. A primal dual interior point algorithm with an exact and differentiable merit function for nonlinear programming. Optimization Methods Software 14, 136.

Bertsekas, D.P., 1995. Nonlinear Programming. Athena Scientific, Belmont, MA.

Brown, D.J., DeMarzo, P.M., Eaves, B.C., 1996a. Computing zeros of sections of vector bundles using homotopies and relocalization. Mathematics of Operations Research 21, 2643.

Brown, D.J., DeMarzo, P.M., Eaves, B.C., 1996b. Computing equilibria when asset markets are incomplete. Econometrica 64, 127. 
DeMarzo, P.M., Eaves, B.C., 1996. Computing equilibria of GEI by relocalization on a Grassmann manifold. Journal of Mathematical Economics 26, 479497.

Duffie, D., Shafer, W., 1985. Equilibrium in incomplete markets I. Journal of Mathematical Economics 14, 285300.

Esteban Bravo, M., 2004. Computing equilibria in general equilibrium models via interior point methods. Computational Economics 23, 147171.

Garcia, C.B., Zangwill, W.I., 1981. Pathways to Solutions, Fixed Points, and Equilibria. Prentice Hall, Englewood Cliffs, NJ.

Gill, P.E., Murray, W., Sauders, M.A., Tomlin, J.A., Wright, M.H., 1986. On projected Newton barrier methods for linear programming and an equivalence to Karmarkar's projective method. Mathematical Programming 36, 183209.

Hart, O.D., 1975. On the optimality of equilibrium when the market structure is incomplete. Journal of Economic Theory 11, 418443.

Hens, T., 1998. Incomplete markets. In: Kirman, A. (Ed.), Elements of General Equilibrium Theory, Festschrift in Honor of Gérard Debreu. Oxford University Press, Oxford, pp. 139210.

Heinkenschloss, M., Ulbrich, M., Ulbrich, S., 1999. Superlinear and quadratic convergence of affine scaling interior point Newton methods for problems with simple bounds without strict complemen tarity assumption. Mathematical Programming 86, 615635.

Herings, P.J. J., Kubler, F., 2002. Computing equilibria in finance economies. Mathematics of Operations Research 27, 637646.

Kubler, F., 2001. Computable general equilibrium with financial markets. Economic Theory 18, 7396.

Kubler, F., Schmedders, K., 2000. Computing equilibria in stochastic finance economies. Computational Economics 15, 145172.

Magill, M.J.P., Quinzii, M., 1996. Theory of Incomplete Markets, vol. 1. MIT Press, Massachusetts.

Magill, M.J.P., Shafer, W., 1991. Incomplete Markets. In: Hildenbrand, W., Sonnenschein, H. (Eds.), Handbook of Mathematical Economics, vol. IV. North Holland, New York, pp. 15231614.

Nocedal, J., Wright, S.J., 1999. Numerical optimization. Springer Series in Operations Research. Springer, New York.

Radner, R., 1972. Existence of equilibrium of plans, prices, and price expectations in a sequence of markets. Econometrica 40, 289303.

Schmedders, K., 1998. Computing equilibria in the general equilibrium model with incomplete asset markets. Journal of Economic Dynamics and Control 22, 13751401.

Schmedders, K., 1999. A homotopy algorithm and an index theorem for the general equilibrium model with incomplete asset markets. Journal of Mathematical Economics 32, 225241.

Yamashita, H., 1998. A globally convergent primal dual interior point methods for constrained optimization. Optimization Methods \& Software 10, 443469.

Yamashita, H., Yabe, H., 1996. Superlinear and quadratic convergence of some primal dual interior point methods for constrained optimization. Mathematical Programming 75, 377397.

Watson, L.T., 2000. Theory of globally convergent probability one homotopies for nonlinear programming. SIAM Journal of Optimization 11 (3), 761780. 